Jelena Issajeva

Tallinn University of Technology,

Estonia

Akadeemia tee 3, 12611 Tallinn, Estonia

E-mail: jelena.issajeva@gmail.com

Ahti-Veikko Pietarinen

Tallinn University of Technology, Estonia

Akadeemia tee 3, 12611 Tallinn, Estonia

\title{
THE HETEROGENOUS AND DYNAMIC NATURE OF MENTAL IMAGES: An empirical study
}

\begin{abstract}
This article addresses the problem of the nature of mental imagery from a new perspective. It suggests that sign-theoretical approach as elaborated by $C$. S. Peirce can give a better and more comprehensive explanation of mental imagery. Our empirical findings follow the methodology of cognitive semiotics and they show that (i) properties of mental images are heterogenous in nature; (ii) properties of mental images are dependent on the characteristics of object-stimulus; (iii) properties of mental images are dependent on individual differences in imaginary capacities. This suggests that, contrary to representational accounts, mental imagery is not based on one dominant representational format. Imagery constitutes a complex system of signs consisting of several sign elements and dynamic relations. A sign-theoretical account may give a better explanation of the nature of mental imagery, as it accommodates heterogenous evidence from this experiment.
\end{abstract}

Keywords: mental imagery, representation, experimental semiotics, theory of signs, Peirce.

\section{Introduction}

Since the 'cognitive revolution' the question about the nature of mental imagery (MI) remains one of the most debated ones in cognitive sciences and philosophy of mind. This article presents new empirical evidence on the matter that follows the methodology of cognitive semiotics. The experimental results showed that (i) properties of mental images are heterogenous in nature; (ii) properties of mental images are dependent on characteristics of object-stimulus; (ii) properties of mental images are dependent on individual differences in imaginary capacities. These results conform with empirical data in neuroscience (Bartolomeo 2008; Moro et al. 2008; Dulin et al. 2008), which claim that one dominant representational account does not adequately 
explain MI. Supported by empirical findings, this paper argues that mental imagery is better explained in terms of sign theory as proposed by C. S. Peirce.

What is MI? Traditionally, two dominant rival theories have been proposed, (quasi-)pictorial and propositional. According to the (quasi-) pictorial theory, mental images are picture-like representations in the mind (Kosslyn 1978, 1980, 1994; Pinker and Finke 1980; Finke, Pinker and Farah 1989). Proponents of propositional theory, on the other hand, claim that MI constitutes verbal representations or language-like descriptions (Pylyshyn 1973, 1981, 2002, 2003; Fodor 1975, 1990). The controversy between the two constitutes the Mental Imagery Debate. ${ }^{1}$ It is common to understand both rivals in the framework of computational-representational paradigm ${ }^{2}$ of mind, which implies that all mental states are products of mental computation. In this context, representational theory is focused on a search of one dominant format or $\operatorname{code}^{3}$ that underlies mental imagery, as well as other mental states.

Adherents of both representational theories have deployed empirical methods to prove their respective claims. Kosslyn and colleagues have experimentally shown that mental imagery (MI) has certain spatial and picture-like properties (size, colors, shapes, dimensions, distances, etc.) and thus concluded that images are most likely pictorial representations (Kosslyn 1980, 1988, 1994; Kosslyn et al. 2006; Shepard and Metzler 1971; Pinker and Finke 1980; Shepard and Cooper 1982; Slotnick et al. 2005). In contrast, Pylyshyn and colleagues (Fodor 1975, 1990; Slezak 1990, 1991, 1995) argued that there is substantial empirical evidence to think of images as being descriptions formulated in language(-like) terms rather than pictures (see for example Fodor's "Language of Thought" hypothesis, 1975).

Despite persistent ambiguities of data on the matter, Imagery Debate was claimed to be solved in favor of (quasi-)pictorial theory of MI (Kosslyn 1994; Pearson and Kosslyn 2015). However, in the light of new experimental methods and results the previous long-standing theories of MI have been reconsidered. Research shows that there are significant difficulties in the representationalist (either pictorial or propositional) approach, some of which are inherent to the representational-computational paradigm (Milikan 1984; von Eckardt 1993;

1 On the Imagery Debate and details on the theories of MI, see Thomas 2010, 2014; Kosslyn 1980, 1988, 1994; Kosslyn et al. 2006; Pearson and Kosslyn 2015; Pylyshyn 1973, 1981, 2002, 2003; Tye 1991.

2 On representational-computational paradigm of mind see Van Gelder 1995; Clapin 2002; Marr 2006.

3 The discussion of whether there are two mental codes that underlie our mental states is called dual-common coding debate. The latter stems from Alan Paivio and his work on memory and learning effects (Paivio 1971, 1986). Noteworthy, dual/common coding debate is different from Imagery Debate (which is also called analog/propositional debate), but has often been confused. Dual-common coding debate focuses on whether we learn and memorize information by using one mental code or another. The analog/ propositional debate, in contrast, investigates the nature of MI. It is the latter, which is the focus of present study. 
Bechtel 1998; Knuutila 2005, 2011). First, under similar experimental settings mental imagery can exhibit (at least) both types of properties - verbal and pictorial (Anderson 1978; Pylyshyn 2002; Ganis 2013). Also, most empirical results on MI yield multiple interpretations (for a detailed discussion of explanations of the experimental results, see Pylyshyn 2002). Most replicated experiments on MI often show differing results (Pylyshyn 1981, 2002; Slezak 1990, 1991; Chambers and Reisberg 1985; Rock, Wheeler and Tudor 1989). There is also a significant amount of empirical evidence proving the existence of motor, tactile, auditory properties of mental imagery (for details, see Lacey and Lawson 2013; Keller 2012; Pascual-Leone et al. 1995; Plessinger 2007; Richardson 1995; Gregg and Clark 2007; Schimdt et al. 2014). All this yields to the conclusion that current empirical data cannot be fully accommodated either by (quasi-)pictorial or by propositional accounts of MI. Maybe understanding of mental imagery cannot be restricted to the dichotomy verbal-pictorial and the search of one dominant format ${ }^{4}$ of $\mathrm{MI}$ is misleading. What is mental imagery really like? Is it a picture in the brain, some propositional or verbal string of language-like characteristics, or something else still?

Most of the novel approaches have emerged in this context, such as enactive and attention-based quantification theories. ${ }^{5}$ Both attempt answering the question of what the true nature of mental imagery is (Thomas 2010; Sima 2011). According to the enactivist approach, mental imagery is a mental capacity of an active cognitive search of information in the absence of the actual perceptual stimulus (Thomas 2009: 454-455). Although enactivism is a representational account, it encounters problems such as vagueness of explanation of the nature of MI and inability to explain deep complexity and multiplicity of properties of images. Yet another alternative account - attention-based quantification theory - explains imagery in terms of attentional processes that quantify spatial and visual information by operating upon two working memory structures, namely Qualitative Spatial Representation (QSR) and Visuo-spatial Attention Window (VSAW) (Sima 2011: 2880). The attention-based quantification theory tries to integrate memory and attention to explain MI, but it relies on qualitative representations and encounters the same difficulties as other representational theories. In sum, increasing diversity of alternative theories has not solved the question.

4 By the term "representational format" we mean internal structure of the mental image, or its "cognitive architecture". We use the term "cognitive architecture" largely in the sense of Z. Pylyshyn (2002), namely to mean the underlying structure of MI, that is "properties and mechanisms [that] are intrinsic to, or constitutive of having and using mental images" (Pylyshyn 2002: 159, original emphasis). Noteworthy that we do not take "representational format" to mean phenomenal modality.

5 Alternative theories - enactivism and attention based quantification theory - are relatively unpopular views in solving the issues of MI. Dominant accounts remain representational. Thus, current empirical study was designed to test the consistency of most dominant representational accounts on MI. 
This article suggests a novel semiotic approach to address the question. In particular, it argues that Peirce's theory of signs as proposed applies well to the analysis of mental imagery and that it can give a coherent explanation of diverse empirical data. We begin with a brief analysis of the theoretical premises of the theory of signs as contrasted with traditional representational accounts. Section 3 describes the experimental design, hypotheses, experimental methodology and procedure. Section 4 provides the results of the experiment. Section 5 is the discussion of the results and their analysis.

\section{Theory of signs and MI}

Peirce's theory of signs (or semeiotic) is an account of signification, reference and meaning (Pietarinen 2015). There are several formulations of theories of signs (see for example Saussure 1983; Morris 1938, 1946, 1964), but Peirce's account is distinctive for its "breadth and complexity" (Atkin 2017: 1). It interprets $\mathrm{MI}^{6}$ as a complex system of signs, which consists of three elements - representamen, object and interpretant - and is characterized by dynamic and flexible relations between these elements (Issayeva 2015; Pietarinen 2012). Such an approach begins with the premise that the mind is of a signifying nature. In particular, the human mind is a sign-producing and sign-interpreting system, characterized by the semiotic processes of signification, i.e. by dynamic, changing and context-dependent processes that create signs and manipulate them. Peirce associates cognition with signs. According to Peirce, all our mental states are signs: "we think only in signs" (Peirce 1994, CP 2.302) "a theory of experience, a theory of consciousness" (Zeman 2014: 1). The human mind constitutes "a historically existing continuum of interpretants (which are signs)", i.e. the the history of signification of objects in one's mind (Zeman 2014: 2). Peirce's famous claim was that "man is a sign" (Peirce 1994, CP 5.314; Peirce 1998, EP 1:54), a person consists of her own thinking, and since all thoughts are in signs, a person is a historical series of signs.

But what does it mean to say that MI is a sign system? First, mental imagery has a signifying nature. MI shares the same structure and features with a sign in the human mind. Just as a sign is defined as "something which stands to somebody for something in some respect or capacity" (Peirce 1994, CP 2.228) in the same way a MI can be legitimately characterized. Thus, just as a sign, $\mathrm{MI}$ is comprised of three main elements: representamen, object and interpretant (Peirce 1994, CP 2.228; Peirce 1998, 478). MI also has a

6 Noteworthy that sign-theoretical definition of MI is twofold. According to Peirce imagery can be understood 1) in a narrow sense meaning the representamen element or something that stands for something, or 2) in a broader sense meaning a mental entity consisting of three elements and constituting a signifying whole, i.e. a system of signs. We take mental imagery as a faculty to mean the second. Though, both definitions can be met in the discussion. 
representamen, which is an element that stands for some object or event. MI has necessarily an object, which it signifies. And it has an interpretant or the meaning that holds between representamen and its object. Hence, every mental image signifies something.

Second, to say that mental imagery is a sign system means that MI is guided by dynamic, context-dependent signifying relations between its elements. According to the sign-theoretical account, relations between elements of the sign are dynamic, i.e. they continuously develop and change their characteristics dependent on various factors. As Floyd Merrell (2001) puts it: "signs simply cannot stand still" (Merrell 2001: 37). Similarly, mental images are not stable or fixed, but are rather of dynamic nature. MI evolve and continuously develop under the influence of both internal (e.g. subjective memory, experience and dispositions) and external (e.g. changes in language, objects' features and new knowledge) factors. The latter entails that mental images are dependent on the context, where they were produced, as well as on the subject, who produced or interpreted an image. The changes in the environment significantly influence both the relations and characteristics of the MI elements. Shortly, context as well as personal experience and cognitive dispositions matter. These influence the whole process of signification. Signtheoretical account as proposed by Peirce can accommodate these features. The dynamics, openness and flexibility of the triadic relations allows to explanation of divergent and changing properties of images under the umbrella of one sign-theoretical account.

To sum up, a sign-theoretical approach towards the explanation of mental imagery yields that a mental imagery is a sign system, which consists of three main relata - a signifying-vehicle or representamen, an object and an interpretant - and is characterized by dynamic, context-dependent semiotic relations between them. Together they - a set of sign-elements and semiotic relations - constitute an interconnected network that works together as a whole. Schematically, these assumptions can be depicted in the following way:

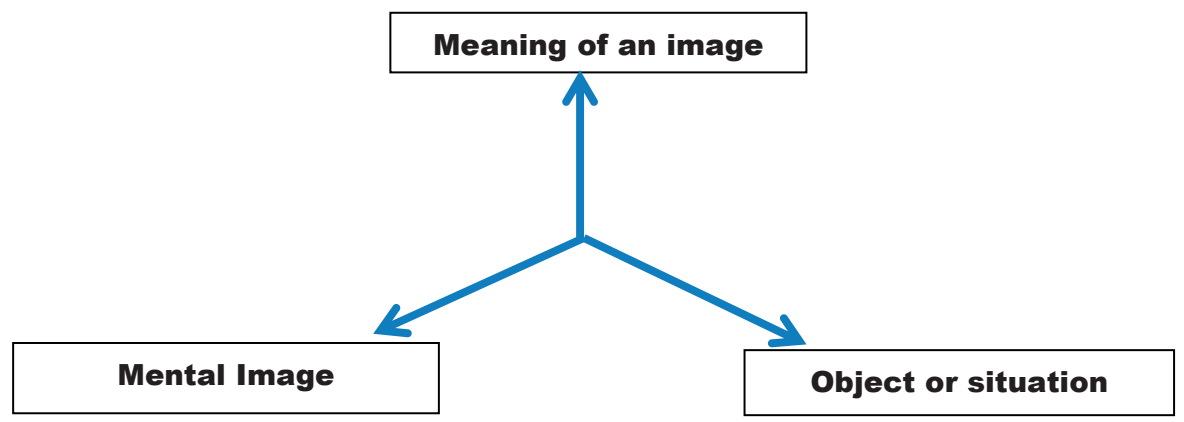

Figure I: Structure of a mental image 
Based on the triadic structure of a sign, Peirce elaborated a fine-grained classification of signs. Each of the three sign elements - representamen, object and interpretant - can be further analysed and divided into three subtypes (Peirce 1994, CP 2.243). In relation to representamen a sign can be divided into Qualisigns, Sinsigns and Legisigns, in relation to its object to Icons, Indexes and Symbols, and finally the third division, Rhemes, Dicisigns and Arguments, is related to the analysis of interpretant (Peirce 1994, CP 2.244-2.252). Together these three trichotomies give rise to ten classes of signs. Applied to MI, classification guides a comprehensive investigation of a mental image and each of its relata, and makes Peirce's semiotics a promising method for an in-depth analysis of MI.

A sign-theoretical approach, thus, might provide a new perspective to MI. It can potentially overcome traditional controversies and limits of the representational accounts and might eventually give a sound and coherent explanation of the MI and its relata.

\section{Methods}

How to test these assumptions? How can we prove that mental imagery indeed can be legitimately interpreted as a sign system?

To test this, we have conducted a test using the methodology of cognitive semiotics and experimental philosophy. The choice of the method of investigation was not arbitrary. First, semiotics is the science that studies signs and their use. In particular, methods of experimental cognitive semiotics and examples of cognitive task design offer a unique way to test the production of a sign in its dynamics and track the most fundamental features of the signification process.

Second, MI constitutes a complex theoretical and interdisciplinary problem with a long tradition in philosophy of mind. Thus the research methods from philosophy sharpen the theoretical hypotheses and our experimental design. In particular, the methodology of experimental philosophy was employed to empirically investigate MI. Experimental philosophy approaches philosophical problems from empirical perspectives. This is of a special value in approaching the debate about MI.

Finally, experimental methods ${ }^{7}$ conform to the investigation of imagery in cognitive psychology. The latter has resulted in divergent empirical data

7 Although current study uses mostly qualitative research methods and does not rely on the brain scanning techniques (fMRI and EEG), the results of our study are still taken to be contributive to the discussion of MI. There are a series of high-powered qualitative research that gives important results on the functions of images without brain scanning and physiological response potential techniques. Actually, some of the classic and most influential experiments on MI (e.g. Perky 1910; Shepard and Metzler 1971; Paivio 1971) are qualitative in nature. Besides, traditional representationalist experimentations on MI (mental scanning, mental mapping and mental paper folding), while based on EEG or 
regarding the nature of $\mathrm{MI}$. Hence, these experimental methods seem appropriate and they take into consideration the relevant previous research. Thus, to show the applicability of the sign-theoretical approach towards the investigation of $\mathrm{MI}$, the relevance of its results to the understanding of the nature and function of MI, as well as its correspondence to the previous research, the experimental method was chosen as the most suitable way to test whether mental imagery shares the same characteristics with a sign.

The experimental design is based on the standard methods and cognitive tasks used in cognitive semiotics and in experimental philosophy. The experimentation began with the short introductory pre-test questionnaire to check the statistically relevant information about age, nationality, cultural and educational backgrounds of the participants. The pre-test was followed by an actual experiment that consisted of three different cognitive tasks. An experiment was finished by the Psi-Q after-test (The Plymouth Sensory Imagery Questionnaire).

The latter constitutes a well-known test on evaluation of imaginary capacities - its vividness and intensity - that was elaborated by psychologists at Plymouth University (Andrade et al. 2013). The essential advantage of the Psi-Q test as compared to other similar questionnaires ${ }^{8}$ is its sensitivity to images across a wide range of modalities: vision, sound, smell, taste, touch, bodily sensation and emotional feeling. This allows to test individual differences in imaginary capacities in more detail. For this reason the Psi-Q test was chosen to measure individual imagery capacities. Finally, the data gathered was analysed using the methods of descriptive statistics -SPSS and R-studio digital services.

\subsection{Experimental hypotheses}

Based on the theoretical premises of a theory of signs the following experimental hypotheses were formulated. The main theoretical hypothesis is that mental imagery can be legitimately viewed as a system of signs:

1) MI shares the same structure with a sign. In other words, MI has an object, interpretant and representamen.

2) $\mathrm{MI}$ is formed in a semiotic process, i.e. inside a network of the signifying relations. The relations between MI's elements define the particular properties of the final image produced.

fMRI, also rely on qualitative methods, including introspection and self-reports. Thus, we believe that our choice of experimental methodology is justified and conforms both to the standards and the practice of the methodological choices used to investigate MI.

8 There are several tests to evaluate vividness of MI: Betts Questionnaire upon Mental Imagery (QMI; Betts 1909; Sheehan 1967), Marks' Vividness of Visual Imagery Questionnaire (VVIQ and VVIQ2; Marks 1973), Gordon's Test of Visual Imagery Control (TVIC; Gordon 1949). The Psi-Q test was chosen before other alternatives, because it allows to evaluate not only the visual MI, but vivdness of images across all sense-modalities. 
To test these the triadic structure of an image was manipulated to uncover the potential correlation between the properties of imagined object and the properties of the final image produced. As a result theoretical sub-hypotheses 1 and 2 were simplified into the following experimental hypotheses:

H0: Mental image has the same or similar characteristics, regardless of the characteristics of an object.

Ha: Mental image has different characteristics. Particular properties of an object influence the characteristics of an image formed to present this object.

In order to analyze these hypotheses, cognitive experimentation was divided into three tasks given to each participant: pictorial, verbal and diagrammatic. Following Peirce's typology of signs, such a task division was chosen to represent distinctive differences in object-stimulus that were supposed to influence a final image. The judgement about statistical significance of the test results will be made on the basis of significance level, the value of which for the sake of the current experiment is taken to be 0.05 (i.e. $\alpha=0.05$ ). The choice of the significance level was guided by the cognitive demands of the experiment: small sample size, equal sample groups, several cognitive tasks and multiple categories of answers. Next, the probability (p-value) that measures the evidence against the null hypothesis, i.e. the probability of either acceptance or rejection of null-hypothesis for the current empirical test is $\mathrm{p} \leq 0.05$.

\subsection{Materials and tasks}

The experiment was designed in the following way: the same object by meaning (that is, by keeping its interpretant fixed) was suggested in three different ways - pictorial, verbal and diagrammatic - to experimental subjects. These three ways of object's presentation refer to Peirce's classification of signs and, in particular, to the three sign types as related to the objects of a sign - icon, index, symbol. An icon has some resemblance with the stimulus, such as something comprehended as a picture. A symbol represents by generality of its objects (such as a convention, language, text). Finally, index represents by causal connections. Hence, diagrams were chosen as the way to introduce causal connections, pictures as a way to represent iconic connections, and language/text to represent symbolic connections respectively. This typology ${ }^{9}$ underlies the three ways in which the object was given in experimental tasks: pictorial, verbal and diagrammatic.

Each experimental task constituted a short story presented either i) pictorially (as a sequence of related pictures, e.g. comics), ii) verbally (written in language story), iii) diagrammatically (as a scheme with arrows and lines). Participants were asked to imagine the rest of the story using any method of expression. The choice of the stories was not arbitrary. Main criteria were the following: a) the story is easy to understand, vocabulary and formulation

9 The same typology was applied to evaluate experimental answers as belonging to pictorial, verbal or diagrammatic categories. 
of the sentences are simple and straightforward to be understood by nonnative speakers with a good command of English; b) the story is concise so that the participants could easily hold in their minds the entire plot; c) the story provokes imagination, i.e. narrative and the plot that it develops is sufficiently interesting for subjects to proceed imagining the end of the story. Stories were written by actual writers, story-tellers and narrators (Chopin 2016; Baum 2016) and were chosen from material similar to children's books, ensuring the points (a)-(c). Considerable attempts were made to have all three modalities (picture, text and diagram) reflect the content of the story as precise as possible, and a professional sketch artist was used for that purpose. In total, there were three different stories presented in each of the three ways.

In addition, the choice of the stories was influenced by semantic differences and cognitive demands. It was important to choose semantically different stories, i.e. those that would put forward a different set of questions in front of the subjects and in this way would suggest different images to be produced as the solution for each of the cognitive task. The existence of such differences was expected to prove one of the sub-hypotheses of the project, namely that mental imagery is task- and context-dependent.

The expected reaction to a story-stimulus is the production of the image that is influenced by the suggested properties of the object - pictorial, verbal or diagrammatic. Thus, the final image is supposed to be different across different cognitive tasks and have distinct similar characteristics within each type of the tasks. An expected result is that the same object (by meaning) expressed in different ways produce different images/representamens.

In sum, the correlation between the image and the object is the target of the experimental investigation. Generally, each experimental task is structured in the following way: the manipulated object (story-stimulus) constitutes the independent variable, while the affected representamen (an image) constitutes the dependent variable. The fixed interpretant (i.e. the same meaning) is the control variable. Schematically, this is depicted as follows:

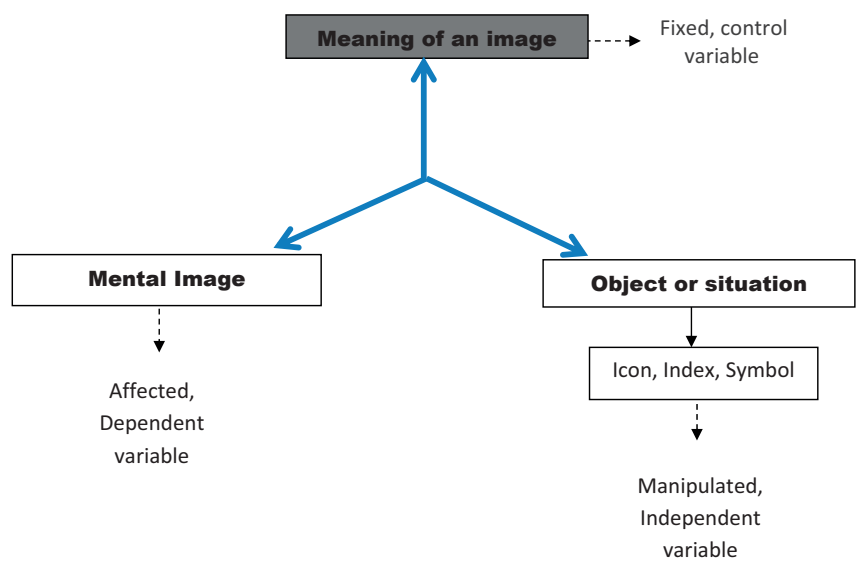

Figure II: Structure of an image experiment 


\subsection{Population and sampling}

The target population of the experiment (i.e. the target sample) was international students of Estonia. ${ }^{10}$ The choice of the target sample was guided mainly by the principle of convenience sampling, but with the several advances. First advantage was internationality of a sample. In current empirical study participated students of 14 different nationalities. Second advantage of sampling was differences in economic backgrounds of participants. Subjects of 20 professions or competencies took part in current study. Such diverse national, cultural and professional backgrounds of participants make them a suitable and available target population to investigate main hypothesis of the research project. Moreover, a target population of international students from various backgrounds might be a good representative of a wider international population.

At all events, the current research professes to be the first step in the investigation of the sign-theoretical account of MI. The sample of the current experimentation consisted of international students of Tallinn University of Technology (TalTech), ${ }^{11}$ mostly bachelor-degree students. The sample size was 40 sampling units, i.e. 40 subjects participated in the experiment. Participants were chosen by the volunteer sampling. Although subjects were not randomly selected, the equal probability of participation was guaranteed by an equal information distribution among all international students via the institute's international office. A complement of free cinema tickets was offered to volunteers. Age of the participants ranged from 18 to 37, whereas an average age of the participants was 26.7 years.

Next, the experimentation was conducted among those international students whose English competence was ascertained to be very good. We asked participants' English language competence in the introductory pre-test setting. It showed that average level of language competence varied between "upper-intermediate (B2)" (42,5\%) and "Advanced (C1)" (32,5\%), which was taken to be sufficient for comprehension of the tasks. $10 \%$ of all participants evaluated their English language competence as "intermediate". Native English speakers constituted 15\% of all participants.

All participants were randomly divided onto two groups, 20 subjects each. Each group received slightly different tasks to avoid the bias of recognizing the purpose of experimentation and to additionally test the potential differences in subjects' performance on different cognitive tasks. Instructions and task formulations were given in English and remained the same across

10 In recent years Estonia has gained significant popularity among international students. Just for the academic year 2015/2016 Estonia has hosted 3800 international degree students, more than 1500 exchange students and ca 400 participants of summer or winter schools. (From http://www.studyinestonia.ee.)

11 Tallinn, as being the capital of Estonia, attracts more international students compared to other cities of Estonia. So, Tallinn University of Technology currently hosts most of the international students in Estonia. For these considerations international students of TalTech were taken as a sample population of the research project. 
experimental groups, but the stimulus of cognitive task differed. The number of answers was: 60 answers in each experimental group (20 subjects solved 3 tasks) and 120 answers in total. This number of answers is assumed to be large enough to show statistical relevance of the answers received and make legitimate conclusions about acceptance or rejection of the null-hypothesis.

\subsection{Procedure}

The experiment took place in an ordinary classroom of TalTech. In order to minimize cognitive bias and to reduce (to the extent possible) the tacit knowledge effect, the experiment was silent, i.e. participants did not know that they are participating in an empirical test, nor did they know the theoretical background or the hypotheses tested. Subjects were invited to help their university's researcher in accomplishing several game-like tasks for her doctorate dissertation. All instructions of the cognitive tasks were given directly by the experimenter before the participant started fulfilling the task. The experimenter made sure that participant understood the task and instructions by receiving a personal confirmation from the participant and answering all the questions (if there were any). During the performance of the task there was no interaction between the experimenter and the subject. The experimentation was conducted in English.

The experimentation began with a series of pilot experiments, which were conducted to check whether subjects correctly understood cognitive task, whether task instructions are clear enough, and whether the order in which tasks are given influences the responses. In total, two pilot investigations were conducted and 63 students participated in the pilot tests. Pilot experiments showed that change of the order, in which cognitive tasks are given, does not influence the responses. Additionally, subjects were sensitive towards the precise formulation of the instructions of the tasks. Thus, the results of the pilot tests helped to sharpen experimental design, formulate instructions in a clearer and more comprehensible way, eliminate the difference in task order and simplify the experiment.

The actual experiment began with the short introductory pre-test questionnaire to provide experimentally important information about participants (age, nationality, educational background, profession or field of study, English competence level). The pre-test questionnaire was followed by the three cognitive tasks given to each participant. Forty participants were divided into two groups (the study and the control groups), and were introduced with the three short stories which were the material on the three cognitive tasks for them to solve (Appendix A). The first task was a pictorial story (a sequence of pictures), second task a verbal one (text), and the third diagrammatic, including both verbal and pictorial elements. The diagram expressed the story via abstract relations (Appendix A). The order of the tasks remained the same across the two groups. By their content, these tasks were 
distributed in the following way: Group 1 received the first story pictorially, the second verbally and the third diagrammatically. Group 2 received the third story pictorially, the first verbally, and the second diagrammatically. Participants were equally instructed on each of the tasks as follows: "a) Look/ Read carefully the story. What will happen next? b) Imagine the rest of the story, and c) Express the imagined on the next page using any method of expression". The experimentation was finished with the qualitative after-test - the Psi-Q test - where participants were invited to evaluate the "subjective vividness" of their imagery capacity.

In total, there were three stories or stimuli presented via three different modalities, totalling six tasks, with the same instructions on how to solve them. The response time was approximately 30 minutes (no sharp time constraints were given to eliminate anxiety etc.). The answers were expected to differ on various stimuli within each group and to coincide on similar stimuli across the two groups.

The experimentation was fomulated and manipulated in this way in order to be able to show that a difference in the initial traits of the imaginary object - pictorial, verbal and diagrammatic - influences "the sign" that represents this object in the final image. To minimize the tacit knowledge effect among experimental subjects and to account for cognitive biases concerning understanding the theoretical background, the content of the stories differed across three tasks within each of the group, while the stories were the same by their content across experimental groups. This ensured that "the interpretant" of the final image was fixed and repeated across experimental groups.

The after-test (Psi-Q test) was then assumed to reveal individual differences in the imaginary abilities of the subjects. That test would check for a correlation between individual imaginary capacity and the response type across three cognitive tasks. We expected those participants who estimate their scores to be high on the vividness of their MI to use a more detailed iconic imagery, while subjects with a lower vividness scores would use more symbolic or abstract imagery.

\section{Results}

The results of the experiment were evaluated in a categorical (nominal) scale that reflects the type of answer participants chose to produce as their final image on each of the three cognitive stimuli. There were thus three general categories: pictorial, verbal and diagrammatic. The reason for the choice of the method of classifying responses in this way ${ }^{12}$ comes from the

12 Noteworthy, according to Peirce's theory, there can hardly be found "pure signs", i.e. the features (symbolic+indexical, indexical+iconic etc.) are often occur to be mixed in signs. This does not preclude us to evaluate responses, following his typology of signs, as belonging to three general categories: pictorial, verbal, diagrammatic (as described 
theoretical framework of Peirce's typology of signs, which corresponds to the three ways in which the objects of the stimuli are presented, namely iconic, symbolic, indexical, in the formulation of the three cognitive tasks.

The classification of the responses was made using the following reasoning: a) If the imaginary answer resembled its respective stimulus, then it corresponded to an iconic sign and was categorized as 'pictorial'. b) If the final image expressed generality (i.e. is a symbol), then it was classified as 'verbal'. c) If the produced image represented some causal connections (i.e. index), then it was labeled 'diagrammatic'. In this way, all three categories of answers conform to the theory as well as to the demands of the study design.

The responses of the experiment were distributed in the following way: for pictorial stimulus (Task 1) 15 answers out of 40 were given pictorially (37,5\% of all respondents). For the same task, 22 answers were verbal and 3 diagrammatic ( $55 \%$ and $7,5 \%$, respectively). It is noteworthy that altogether 18 answers out of 40 were given in a non-verbal way (i.e. pictorial and diagrammatic), which constituted $45 \%$ of all answers.

Table II: Answers for pictorial stimulus (Task 1).

\begin{tabular}{|c|r|r|}
\hline Method/Frequency & Frequency & Percent \\
\hline Diagr & 3 & 7,5 \\
Pictor & 15 & 37,5 \\
Verbal & 22 & 55,0 \\
Total & 40 & 100,0 \\
\hline
\end{tabular}

Next, for verbal stimulus (Task 2) 4 subjects out of 40 answered pictorially (10\% of all respondents). For the same task we received 31 verbal answers and 5 diagrammatic ( $77,5 \%$ and $12,5 \%$ of all respondents, respectively). The total number of non-verbal answers were the lowest among all three cognitive tasks, namely 9 answers (22,5\% of all respondents).

Table III: Answers for verbal stimulus (Task 2).

\begin{tabular}{|c|r|r|}
\hline Method/Frequency & Frequency & Percent \\
\hline Diagr & 5 & 12,5 \\
Pictor & 4 & 10,0 \\
Verbal & 31 & 77,5 \\
Total & 40 & 100,0 \\
\hline
\end{tabular}

Finally, for diagrammatic stimulus (Task 3) we received 4 pictorial, 22 verbal and 14 diagrammatic answers $(10 \%, 55 \%$ and $35 \%$ of all respondents, respectively). Similarly to the answers for Task 1 , we found that the total amount of non-verbal answers was quite high: 18 answers out of 40, that is $45 \%$ of all repsondents.

above) by the most dominant/prevalent feature of the answer (i.e. either iconic, indexical or symbolic). 
Table IV: Answers for diagrammatic stimulus (Task 3).

\begin{tabular}{|c|r|r|}
\hline Method/Frequency & Frequency & Percent \\
\hline Diagr & 14 & 35,0 \\
Pictor & 4 & \\
10,0 \\
Verbal & 22 & 55,0 \\
Total & 40 & 100,0 \\
\hline
\end{tabular}

No significant difference between two experimental groups and the type of the answer was found. For this reason, all results were evaluated together. The general distribution of answers across all three categories can be seen in Table IV and in the corresponding graph in Figure III:

Table V: Frequencies of answer distribution

\begin{tabular}{|l|r|r|r|}
\hline Method/Task & \multicolumn{1}{|l|}{ Pictorial } & Verbal & Diagrammatic \\
\hline Diagrammatic & 3 & 5 & 14 \\
\hline Pictorial & 15 & 4 & 4 \\
\hline Verbal & 22 & 31 & 22 \\
\hline Total & 40 & 40 & 40 \\
\hline
\end{tabular}

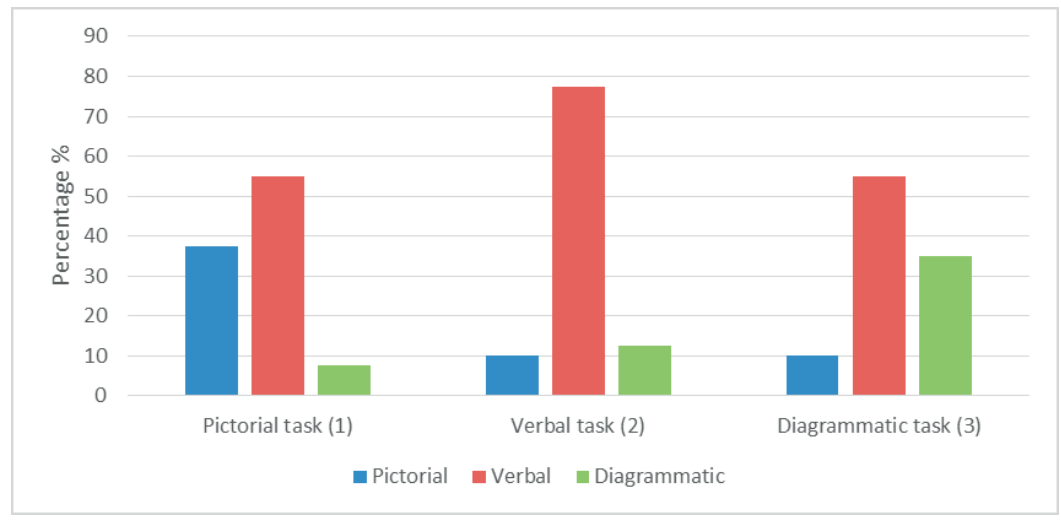

Figure III: Distribution of answers in percentage

The methods of descriptive and inferential statistics were used to analyze these results. In particular, statistical programs SPSS and R-studio were both used for statistical analysis and relevant calculations.

\section{Discussion}

What do these results show? Can we confirm or deny the initial hypothesis? The Pearson's Chi-squared test ${ }^{13}$ was performed to calculate the p-value and to examine whether there is a significant relation between

13 Pearson's Chi-squared test was chosen due to demands of current experimental design, since it allows evaluating several sets of categorical data. 
properties of an object and those of an image. The R-studio statistical calculations showed $\mathrm{X}^{2}(\mathrm{~N}=40)=22,045 ; \mathrm{p}=0,0001963$ with $\mathrm{df}$ (degree of freedom $)=4$. To check and confirm these calculations, we applied Fisher's test using R-studio program. The Fisher's test showed slightly different $p$-value, $p$ $=0,0004802$. However, the results of both tests confirmed that the relation between two variables (the method of response and the type of the task) was strongly significant, with $\mathrm{p}<0,01$. Similar calculations were performed using SPSS. It confirmed previous findings with $\mathrm{X}^{2}(\mathrm{~N}=40)=22,045 ; \mathrm{p}=0,0001963$. Our results suggest that the null-hypothesis, namely that the characteristics of mental images remain the same regardless of the characteristics of its object, should be rejected.

The low $\mathrm{p}$-value $(\mathrm{p} \leq 0.05)$ confirms the alternative hypothesis, which proposes that there is a significant interrelation between properties of an object and properties of an image. Particular properties of an object influence the characteristics of an image formed to present this object. It can be seen from Tables I-III that the distribution of answers across three types of tasks was heterogeneous. In particular, the largest number of pictorial responses $(37,5 \%)$ was given on pictorial stimulus (Task 1). Similar observations hold for verbal and diagrammatic answers. Stimulus influences the formation of mental image significantly. This leads to the conclusion that mental imagery does not share certain characteristics that would be independent of the characteristics of its object. On the contrary, various properties of the object evoke various images. This challenges the idea that one cognitive format underlies the formation of mental images.

At the same time, we can observe from Tables I-III that all three response types (pictorial, verbal and diagrammatic) were used to solve the three tasks. Heterogeneity of how answers were distributed confirms the hypothesis that mental imagery cannot be understood from the perspective of one type of mental format or representation. Subjects tend to choose various methods for their image-formation that varies with multiple influencing factors. This conforms to the sign-theoretical account, as according to it there cannot be pure images of some particular type. Signs are subtle combinations of their elements and dynamic relations between them. Thus, any image might have several (that is, symbolic, iconic, indexical) ${ }^{14}$ characteristics simultaneously. Such heterogeneity is clearly seen from the distribution of the answers in the experiment.

14 The triadic division on icons, symbols and indices refers to Peirce's classification of signs (Peirce 1994, 1998). The explanation of the theoretically important elements of the classification is presented in another paper. In brief, a sign is an icon if it has a power to signify its object doe to a similarity with that object, an index if it has a power to signify its object due to a real relation with the object of its signification, and a symbol if it has a power to signify its object to an interpreter solely because it will be so interpreted. A sign can be an icon and an index simultaneously, and nothing real can be a pure icon or a pure index. Likewise, a sign can be a symbol, an icon, and an index simultaneously. 
In addition, the tendency towards the mixture of answer-types was confirmed by an observational part of the study, namely the experimenter's interaction with the participants. While receiving the instructions on how to solve the tasks, several students asked whether they can use the mixture of several methods. Since this question occurred frequently, we concluded that they were inclined to use multiple modes of imagining. This could be seen as a confirmation that there is no one unified format underlying MI. Also the answers indicate this tendency towards the mixture of the response-types and the characteristics. Under a closer investigation, it occurred that students tended to use (at least) some mixture of answer-types. For example, while giving a pictorial answer to the imagined stimulus, a participant might have used arrows (diagrammatic method) to show the order of the pictures drawn; sometimes there were small linguistic 'clouds' indicating a direct speech etc. Observations did not conflict with the interpretation of the experimental data and can also be read as confirming the main hypothesis, that mental imagery, as signs, has different characteristics. Particular properties of MI are influenced by multiple factors, including characteristics of the objectstimulus, task demands, the context as well as individual differences. In brief, there is no dominant format underlying MI.

Next, we assumed that individual differences influence the imageformation. To analyze this two tests were conducted. An introductory pretest checked whether individual variations in native language, cultural background or occupation influence mental imagery. The after-test (Psi-Q test) evaluated subjective vividness of imagery capacity and its influence on the response type. In particular, we checked whether a) participants across different backgrounds answered similarly ( $\mathrm{HO})$ or differently (Ha). For the Psi-Q test, we tested whether b) all participants, regardless of any subjective differences in the vividness of images, answer similarly (H0) or differently (Ha). The same programs (SPSS and R-studio) were used to statistically analyse the results. The significance level was $\alpha=0.05$. For the first test we found no significant correlation between occupation and response type; $\mathrm{X}^{2}(\mathrm{~N}=40)=2,853 ; \mathrm{p}=0,415$ (with $\mathrm{df}=3$ ); according to Fisher's test this was $\mathrm{p}=0,513$. Since the $\mathrm{p}$-value was over $0.05, \mathrm{H}_{0}$ should be accepted. We interpret this as participants answering similarly to the three cognitive tasks independently of differences in their professions and cultural backgrounds.

Interestingly, for the second test (Psi-Q test) we found a strong correlation between individual differences in MI's vividness and response type. Analysis showed that subjects with higher vividness of MI tended to answer pictorially, producing detailed and elaborated images, whereas subjects with a lower vividness of MI tended to answer verbally, i.e. in a more abstract and general way. The significance level for this test was $\mathrm{p}=$ 0,004 , which confirms the alternative hypothesis, namely that participants answer differently depending on subjective differences in the vividness of images. Our interpretation is that individual differences in cognitive capacities influence the formation of MI. 
Taken all the above into account, a couple of general conclusions concerning the nature of mental imagery may be drawn. First, our empirical research suggests $\mathrm{MI}$ to exhibit characteristics that varies with multiple factors, and thus appears to be heterogeneous in nature. Our proposed interpretation is that MI would be poorly understood assuming it to be of some general or universal mental kind or format. Second, properties of mental images vary with the characteristics of the object-stimulus. MI does not share characteristics independent of the properties of an object-stimulus; rather, MI encapsulates properties of the imagined stimulus, which suggests that features of mental image depend on features of an object that it professes to represent. Finally, properties of mental images are dependent on individual differences in imaginary capacities. Indeed, human cognitive capacities surely should not be assumed to be equal: having more or less vivid imageries is well documented, both within a person and across people. Personal capacities and dispositions influence the characteristics of the produced images.

We could read these conclusions to propose that MI cannot be comprehensively explained by the prevailing representational theories that take MI to be, alternatively, matters of quasi-pictorial or propositional representations (Kosslyn 1980, 1994; Pylyshyn 1981, 2002). Our evidence showed that no dominant representational format underlies imagery. In the very least, MI can hardly be viewed as a static mental representation of a fixed particular format, which is implied by computational-repesentational paradigm (Clapin 2002; Marr 2006). Rather images change their characteristics dependent on the context, task, and the features of the objects. Dependence of an imageformation on the characteristics of its co-related elements strongly suggests that MI is not the matter of a static representation, and that dynamic mental activity occurs within the context of the creation of mentally depicted relations.

A coherent account on the nature of MI would explain such features as the heterogeneity of its characteristics, its task-context-object dependence, and the influence differences have on the image formation, among others. It might be difficult to explain all these facts by traditional representational theories of MI. Although, quasi-pictorial theory could easily accommodate pictorial data, whereas propositional theory - verbal data, the explanation of the current results by traditional accounts will still remain partial. The restriction of MI to quasi-pictorial - propositional dichotomy inevitably neglects at least some of the above-stated characteristics of MI. The reason for this might be hidden in the implicit demand of the dominant computational paradigm: the search of the primary code, which would unravel the complex mechanisms of human mind. However, new research methods and empirical data show that above-stated demand might be misleading (von Eckardt 1993; Bechtel 1998; Knuutila 2005, 2011). The results of current empirical study confirm this idea. Similarly, enactivist theory can potentially explain the dynamic relations and task-object dependence, but it could hardly account for divergent characteristics of images. Enactivism lacks a comprehensive explanation of MI's structure and diversity. 
In contrast, the sign-theoretical approach that we have advocated can accommodate heterogeneity of MI's properties, its task-context-object dependence, and individual differences in imaginary capacities within one framework. First, MI can be seen as a sign that consists of three elements: representamen, object and interpretant. Taking MI to be of this triadic structure allows a detailed explanation of the nature and function of images in human cognition. Second, the theory of signs proceeds to take mental capacities to be of signifying nature. This would connect MI with many other cognitive abilities of the human mind, and would explain individual differences and dispositions in the creation of MI. Third, the theory is concerned with the dynamic and open nature of semiotic relations between the three elements of a sign. This allows it to be applied to the explanation of divergent and changing properties of mental images.

Although experimental findings support the theory of sign towards investigation of MI, one might also argue that there are certain weaknesses in experimental studies conducted on MI. First, our three cognitive tasks might evoke different cognitive capacities (e.g., decision-making, creative thinking etc.) as well. How can one be sure that it was MI that was used to solve these experimental tasks? Now the employment of mental images was ensured by the precise and detailed instructions given by the experimenter and by receiving personal confirmation that each participant understood the task. Nevertheless, it is not straightforward to separate MI from other cognitive faculties or to eliminate their influence on the response rate. Also, the design of the task is not free from criticism, especially if compared with previous studies on similar matters. To this we reply as follows. In contrast to standard cognitive tasks testing MI where subjects are asked to memorize some stimulus, our task to imagine the rest of the story is markedly different. It allows testing the production of an image in natural way, such as what people might use in their daily life while planning, thinking, analysing, reasoning or daydreaming. There are in fact indications that two-thirds of our waking life mind is actually wandering and not well controlled or self-controlled by us, the self, by some cognitive agency (Metzinger 2017). In light of mindwandering theories, it is only natural to test the nature of MI in the proposed manner. The MI produced as a response to our cognitive task is not artificial but spontaneous and may in fact be more 'ecologically valid' - subjects were free to choose any method to imagine and were not asked or expected in any way to remember the stimulus.

Further, one could read the results of the study differently, saying that shown heterogeneity of imaginary characteristics might be understood on phenomenal level only, while internal structure of MI remains one and the same. In this case, internal structure of MI is supposed to be hidden and consciously ${ }^{15}$ inaccessible. While current study does not apply brain-scanning

15 According to the sign-theoretic account, there are several levels of conscious access to a mental sign (for details, see Champagne 2018). The 'sub-personal' level corresponds to single sensations and qualities that are registered by the mind, but are not yet attended by 
techniques in demonstrating the underlying difference in neurophysiological terms, it does show the structural difference of the various images produced as a response on different stimuli, which implies the former. If the representational format of MI would indeed be sub-personally and unconsciously one and the same across different images, then the answers to imaginary tasks would be expected to be similar. But this is not the case. Moreover, one would expect one and the same subject to answer similarly to all stimuli, but this was not the case either. One and the same subject typically used various types of images to answer different stimuli. Based on the above-stated data this difference is statistically significant. Thus, we are inclined to conclude that shown heterogeneity of MI's properties is not (just) phenomenal: the difference in the modes of expression of an internal image does say something about subpersonal and unconscious level of image formation.

Finally, the limited sample size and the volunteer sampling method instead of full randomization may be a limitation of the current research, conducted under limited organisational allowances, and random sampling method and larger sample size is suggested for replication.

To sum up, limitations notwithstanding, explanations of MI should not overlook the potential of seeing them as signs. A sign-theoretical approach might overcome some long-standing controversies and limits of the prevailing representational accounts. In particular, the experimental approach suggests a new perspective where divergent properties of mental images come together under the umbrella of a Peircean theory.

\section{Conclusion}

We studied the nature of mental imagery by an experiment in the theoretical context of Peirce's semiotics (the theory of signs). An empirical test was carried out that hypothesised that mental imagery can be accounted for in that theory. According to the theory, $\mathrm{MI}$ is a sign that consists of three relata: representamen, object and interpretant, and it is characterized by dynamic and context-dependent semiotic relationships. To test the hypothesis, an experiment was designed. The analysis of the results showed that 1) the characteristics of mental images are heterogeneous in nature; 2) properties of mental images are dependent on the characteristics of object-stimulus; 3) properties of mental images are dependent on individual differences in imaginary capacities. These results were interpreted to indicate that, contrary to standard representational accounts, MI does not emerge from one dominant

the conscious self. The internal structure of the mind and MI, however, does not change dependent on the level of conscious accessibility. Higher level of conscious access which is the level of current study - is the indicator of the internal (sub-personal/hidden) structure. The sign-theoretic account that we are applying can accommodate these levels within one theoretical framework. 
representational format (such as quasi-pictorial or propositional). Standard representational accounts may fail to provide comprehensive explanations of heterogeneous characteristics of MI and their context dependence. Our study concludes that these features can, however, be explained by Peirce's theory of signs. The results support the idea that MI can be seen as signs. Under that light, MI constitute complex mental phenomena with manifold traits and dynamic, continuously changing relations between its elements. While new empirical investigations that exploit the sign-theoretical approach are needed, this interpretation of the results of the present experiment is also a strong indication that the theory of signs is a viable methodological alternative that accommodates heterogeneous empirical evidence.

\section{Acknowledgments:}

This empirical research was founded by Estonian Research Council scientific grant PUT1305 "Abduction in the Age of Fundamental Uncertainty", 2017. We would like to thank Amirouche Moktefi $(\mathrm{PhD})$ for his valuable advices and constant help, as well as Liisa Kruusamägi - a talented illustrator - for her work and patience. We would like to express a special gratitude to Kristiina Averin - philosopher and psychologist - for her indispensable help with statistical analysis. The authors declare no conflict to interest.

\section{References:}

Andrade, Jackie, May, Jon, Deeprose, Catherine, Sarah-Jane Baugh and Giorgio Ganis. 2013. Psi-Q: the Plymouth Sensory Imagery Questionnaire. British Journal of Psychology 105. 547-563.

Anderson, John R. 1978. Arguments concerning representations for mental imagery. Psychological Review 85. 249-277.

Atkin, Albert. 2013. Peirce's theory of signs. In Edward N. Zalta (ed.), The Stanford encyclopedia of philosophy. https://plato.stanford.edu/archives/ sum2013/entries/peirce-semiotics/. (accessed 15 September 2017).

Bartolomeo, Paolo 2008. The neural correlates of visual mental imagery: an ongoing debate. Cortex 44(2). 107-108.

Baum, Stuart B. 2017. The blue bottle. http://www.stuartstories.com/activities/ bluebottle.html. (accessed 15 September 2017).

Bechtel, William. 1998. Representations and cognitive explanations: assesing the dynamicist's challenge in cognitive science. Cognitive Science 22(3). 295-318.

Betts, George Herbert. 1909. The distribution and functions of mental imagery. Teachers' College Columbia University Contributions to Education 26. 1-99. 
Chambers, Deborah, Reisberg, Daniel. 1985. Can mental images be ambiguous? Journal of Experimental Psychology: Human Perception and Performance 11. 317-328.

Champagne, Mark. 2018. Consciousness and the Philosophy of Signs. How Peircean Semiotics Combines Phenomenal Qualia and Practical Effects. Dordrecht: Springer.

Chopin, Kate. 2017. The pair of silk stockings. http://www.eastoftheweb.com/ short-stories/UBooks/PairSilk859.shtml. (accessed 15 September 2017).

Clapin, Hugh. 2002. Philosophy of mental representation. Oxford: Clarendon Press.

Dulin, David, Hatwell, Yvette, Pylyshyn, Zenon W., Chokron, Sylvie. 2008. Effects of peripheral and central visual impairment on mental imagery capacity. Neuroscience and Biobehavioral Reviews 32. 1396-1408.

Finke, Ronald A., Pinker Steven, Farah, Martha J. 1989. Reinterpreting visual patterns in mental imagery. Cognitive Science 13. 51-78.

Fodor, Jerry A. 1975. The language of thought. Cambridge, Mass.: Harvard University Press.

Fodor, Jerry A. 1990. A theory of content and other essays. Cambridge, Mass.: MIT Press

Ganis, Giorgio. 2013. Visual mental imagery. In Simon Lacey and Rebecca Lawson (eds.), Multisensory imagery, 9-28. Dordrecht: Springer.

Gordon, Rosemary. 1949. Investigation into some of the factors that favour the formation of stereotype images. British Journal of Psychology 39. 156-167.

Gregg, Melanie J. \& Clark, Terry. 2007. Theoretical and practical applications of mental imagery. In Aaron Williamon and Werner Goebl (eds.), Proceedings of the international symposium on performance science 2013, 295-300. Brussels, Belgium: European Association of Conservatoires (AEC).

Issayeva, Jelena. 2015. Sign theory at work: The mental imagery debate revisited. Sign Systems Studies 43(4), 584-596.

Keller, Peter E. 2012. Mental imagery in music performance: underlying mechanisms and potential benefits. Annals of the New York Academy of Sciences. The neurosciences and music IV learning and memory 1252. 206-213.

Knuuttila, Tarja. 2005. Models as epistemic artefacts: toward a nonrepresentationalist account of scientific representation. Dissertation. University of Helsinki.

Knuuttila, Tarja. 2011. Modelling and representing: an artefactual approach to model-based representation. Studies in History and Philosophy of Science 42. $262-271$.

Kosslyn, Stephen M. 1978. Measuring the visual angle of the mind's eye. Cognitive Psychology 10. 356-89. 
Kosslyn, Stephen M. 1980. Image and mind. Cambridge, Mass.: Harvard University Press.

Kosslyn, Stephen M. 1988. Aspects of a cognitive neuroscience of mental imagery. Science 240(4859). 1621-1626.

Kosslyn, Stephen M. 1994. Image and brain: the resolution of the imagery debate. Cambridge, Mass.: MIT Press.

Kosslyn, Stephen M., Ganis, Giorgio, Thompson, William L. 2006. Mental imagery and the human brain. In Qicheng Jing, Mark R. Rosenzweig, Gerry d'Ydewalle, Houcan Zhang, Hsuan-Chih Chen, Kan Zhang (eds.), Progress in psychological science around the world: neural, cognitive and developmental issues, 195-209. New York, NY: Psychology Press.

Lacey, Simon \& Lawson, Rebecca. 2013. Multisensory imagery. Dordrecht: Springer.

Marks, David F. 1973. Visual imagery in the recall of pictures. British Journal of Psychology 64. 17-24.

Marr, David. 2006. Vision. In Jose L. Bermudez (ed.), Philosophy of psychology. Contemporary readings, 385-406. New York and London: Routledge.

Merrell, Floyd. 2001. Charles Sanders Peirce's concept of the sign. In Paul Cobley (ed.), The Routledge companion to semiotics and linguistics, 28-39. New York and London: Routledge.

Metzinger, Thomas. 2017. Why is mind-wandering interesting for philosophers? In Kieran C.R. Fox \& Kalina Christoff (eds.). The Oxford Handbook of Spontaneous Thought: Mind-wandering, Creativity, Dreaming, and Clinical Conditions. New York, NY: Oxford University Press.

Millikan, Ruth G. 1984. Language, thought and other biological categories. Cambridge, Mass.: MIT Press.

Moro, Valentina, Berlucchi, Giovanni, Lerch, Jason, Tomaiuolo, Francesco, Aglioti, Salvatore M. 2008. Selective deficit of mental visual imagery with intact primary visual cortex and visual perception. Cortex 44. 109-118.

Morris, Charles W. 1938. Foundations of the theory of signs. Chicago, IL: The University of Chicago Press.

Morris, Charles W. 1946. Signs, language and behavior. New York, NY: Prentice-Hall.

Morris, Charles W. 1964. Signification and significance: a study of the relations of signs and values. Cambridge, Mass.: MIT Press.

Pascual-Leone, Alvaro, Nguyet, Dang, Cohen, L.eonardo G., Brasil-Neto, Joaquim P., Cammarota, Angel, Hallett, Mark. 1995. Modulation of muscle responses evoked by transcranial magnetic stimulation during the acquisition of new fine motor skills. Journal of Neurophysiology 74(3). 1037-45. 
Paivio, Allan U. 1971. Imagery and Verbal Processes. New York, NY: Holt, Rinehart and Winston.

Paivio, Allan U. 1986. Mental Representations: A Dual Coding Approach. New York, NY: Oxford University Press.

Pearson, Joel \& Kosslyn, Stephen M. 2015. The heterogeneity of mental representation: ending the imagery debate. Proceedings of the National Academy of Sciences of United States of America 112(33). 10089-10092.

Peirce, Charles S. 1994. The collected papers of Charles Sanders Peirce. Charles Hartshorne and Paul Weiss (eds.). Cambridge, Mass.: Harvard University Press. Vols. VII-VIII (1958), Arthur W. Burks (ed.), Cambridge, Mass.: Harvard University Press.

Peirce, Charles S. 1998. The essential Peirce. Selected philosophical writings. Volume 2 (1893-1913). Nathan Houser, Jonathan R. Eller, Albert C. Lewis, Andre D. Tienne, Cathy L. Clark, D. Bront Davis (eds.). Bloomington and Indianapolis: Indiana University Press.

Perky, Mary C. W. 1910. An experimental study of imagination. American Journal of Psychology (21). 422-52.

Pietarinen, Ahti-Veikko. 2012. Peirce and the logic of image. Semiotica (192), 251-261.

Pietarinen, Ahti-Veikko. 2015. Signs systematically studied: Invitation to Peirce's theory. Sign Systems Studies 43(4), 372-398.

Pinker, Steven \& Finke, Ronald A. 1980. Emergent two-dimensional patterns in images rotated in depth. Journal of Experimental Psychology: Human Perception and Performance (4). 21-35.

Plessinger, Annie. 2007. The effects of mental imagery on athletic performance. http://healthpsych.psy.vanderbilt.edu/HealthPsych/mentalimagery.html (accessed 15 September 2017).

Pylyshyn, Zenon W. 1973. What the mind's eye tells the mind's brain: the critique of mental imagery. Psychological Bulletin 80(1). 1-24.

Pylyshyn, Zenon W. 1981. The imagery debate: analogue media versus tacit knowledge. Psychological Review 88. 16-45.

Pylyshyn Zenon W. 2000. Situating vision in the world. Trends in Cognitive Sciences 4(5). 197-206.

Pylyshyn, Zenon W. 2002. Mental imagery: in search of a theory. Behavioral and Brain Sciences 25(2). 157-238.

Pylyshyn, Zenon W. 2003. Seeing and visualizing: it's not what you think. Cambridge, Mass.: The MIT Press.

Richardson, Peggy A. 1995. Therapeutic imagery and athletic injuries. Journal of Athletic Training 30(1). 10-12.

Rock, Irvin, Wheeler, Deborah \& Tudor, Leslie. 1989. Can we imagine how objects look from other viewpoints? Cognitive Psychology 21(2). 185-210. 
Saussure, Ferdinand. 1983. Course in general linguistics. Translated by Roy Harris. London: Duckworth.

Schmidt, Timo T., Ostwald, Dirk, Blankenburg, Felix. 2014. Imaging tactile imagery: changes in the brain connectivity support perceptual grounding of mental images in primary sensory cortices. Neuroimage 98. 216-24.

Sheehan, Peter W. 1967. A shortened form of Betts' questionnaire upon mental imagery. Journal of Clinical Psychology 23. 386-389.

Shepard, Roger N. \& Metzler, Jacqueline. 1971. Mental rotation of threedimensional objects. Science 171. 701-703.

Shepard, Roger N. \& Cooper, Lynn A. 1982. Mental images and their transformations. Cambridge, Mass.: MIT Press.

Sima, Jan Frederik. 2011. The nature of mental images - an integrative computational theory. In Laura Carlson, Christoph Hölscher, Thomas F. Shipley (eds.), Proceedings of the 33rd Annual Conference of the Cognitive Science Society, 2878-2883. Austin: TX.

Slezak, Peter. 1990. Re-interpreting images. Analysis 50(4). 231-243.

Slezak, Peter. 1991. Can images be rotated and inspected? A test of the pictorial medium theory. The Proceedings of the Thirteenth Annual Conference of the Cognitive Science Society. 55-60.

Slezak, Peter. 1995. The 'philosophical' case against visual imagery. In Peter. Slezak, Terry Caelli, Richard Clark (eds.), Perspectives on cognitive science: theories, experiments and foundations, 237-271. Norwood, NJ: Ablex Publishing.

Slotnick, Scott D., Thompson, William L., Kosslyn, Stephen M. 2005. Visual mental imagery induces retinotopically organized activation of early visual areas. Cerebral Cortex 15(10). 1570-1583.

Thomas, Nigel J. T. 2009. Visual imagery and consciousness. In William P. Banks (ed.), Encyclopedia of consciousness, 445-457. Oxford: Academic Press/Elsevier.

Thomas, Nigel J. T. 2010. Imagery and coherence of imagination. Journal of Philosophical Research 22. 95-127.

Thomas, Nigel J. T. 2014. Mental imagery. In Edward N. Zalta (ed.), The Stanford encyclopedia of philosophy. http://plato.stanford.edu/archives/ fall2014/entries/mental-imagery/. (accessed 15 September 2017).

Tye, Michael. 1991. The imagery debate. Cambridge, Mass.: MIT Press.

Van Gelder, Tim. 1995 What might cognition be, if not computation? The Journal of Philosophy 92. 345-381.

Von Eckardt, Barbara. 1993. What is cognitive science? Cambridge, Mass.: MIT Press.

Zeman, Jay. 1977. Peirce's theory of signs. In Thomas A. Sebeok (ed.), A Perfusion of signs, 22-39. Bloomington: Indiana University Press. 
Appendix A: The Examples of cognitive tasks

Task 1: a) Look carefully at the story. What will happen next? b) Imagine the rest of the story, and c) Express the imagined on the next page using any method of expression.
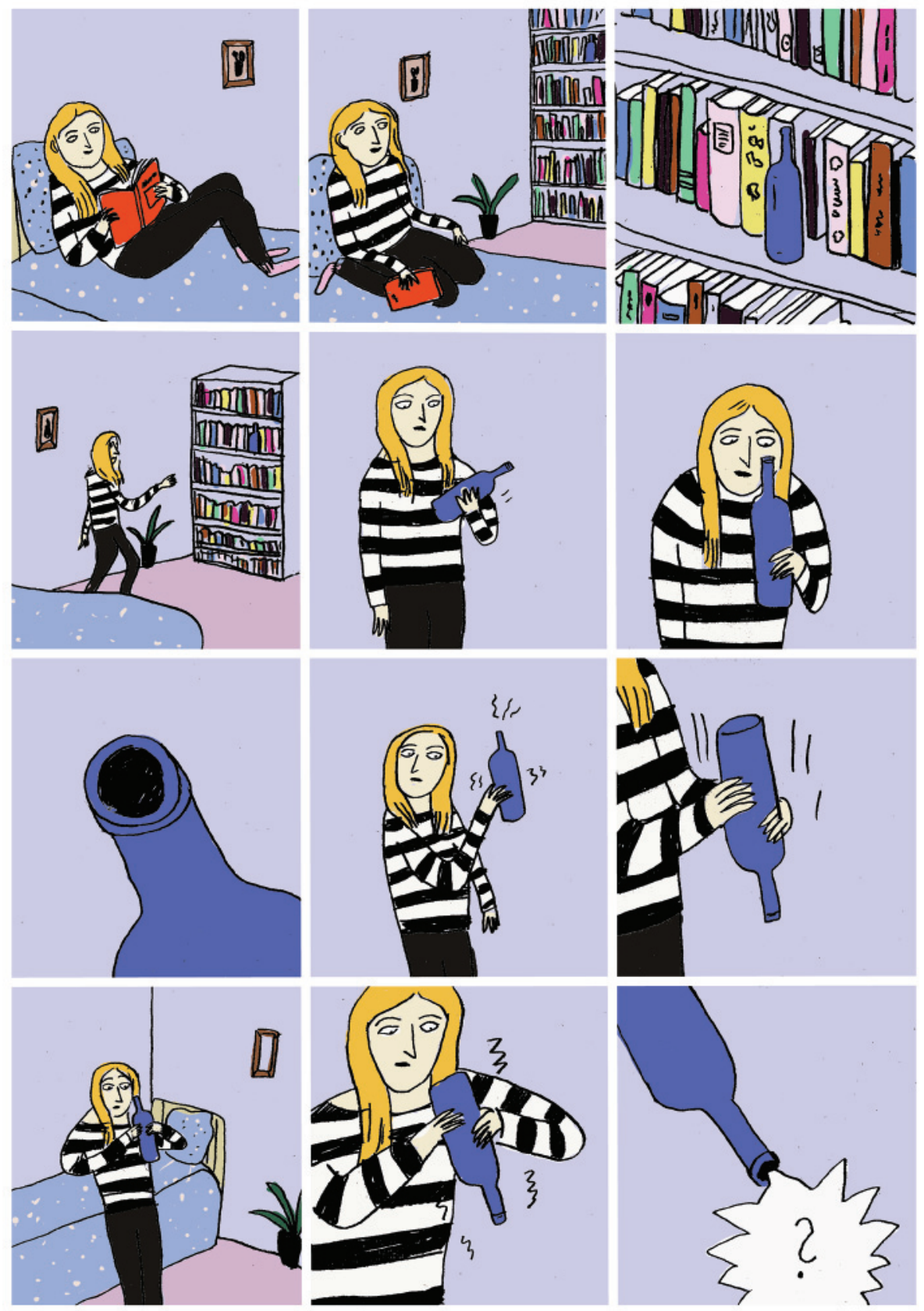
Task 2: a) Read carefully the story. What will happen next? b) Imagine the rest of the story, and c) Express the imagined on the next page using any method of expression.

One night, Millie was up late reading in bed. She finished the book she was reading and looked over to her shelves to see what else she might read before she went to bed. Right there on her shelf was something she had never seen before. It was a blue bottle. The blue bottle was about as tall as a small book, had a round bottom, and a thin neck. And while the bottle looked as if it were made out of glass, Millie could not see through it. Millie got out of bed and went over to the bottle. She picked it up, carefully, afraid that it might break. She was surprised at just how heavy it was. Certainly heavier than any other bottle this size she had ever before lifted.

She looked down into the bottle, but it was too dark inside to see anything. So she shook it. She heard a rattling sound. There was something inside! She turned the bottle upside down and shook it again, to see if anything would fall out. Something almost fell out and then it didn't. Whatever was inside was now stuck in the bottle's neck. Millie shook harder and harder. Finally, something small fell onto the floor. It was a ... 
Task 3: a) Look carefully at the story. What will happen next? b) Imagine the rest of the story, and c) Express the imagined on the next page using any method of expression.
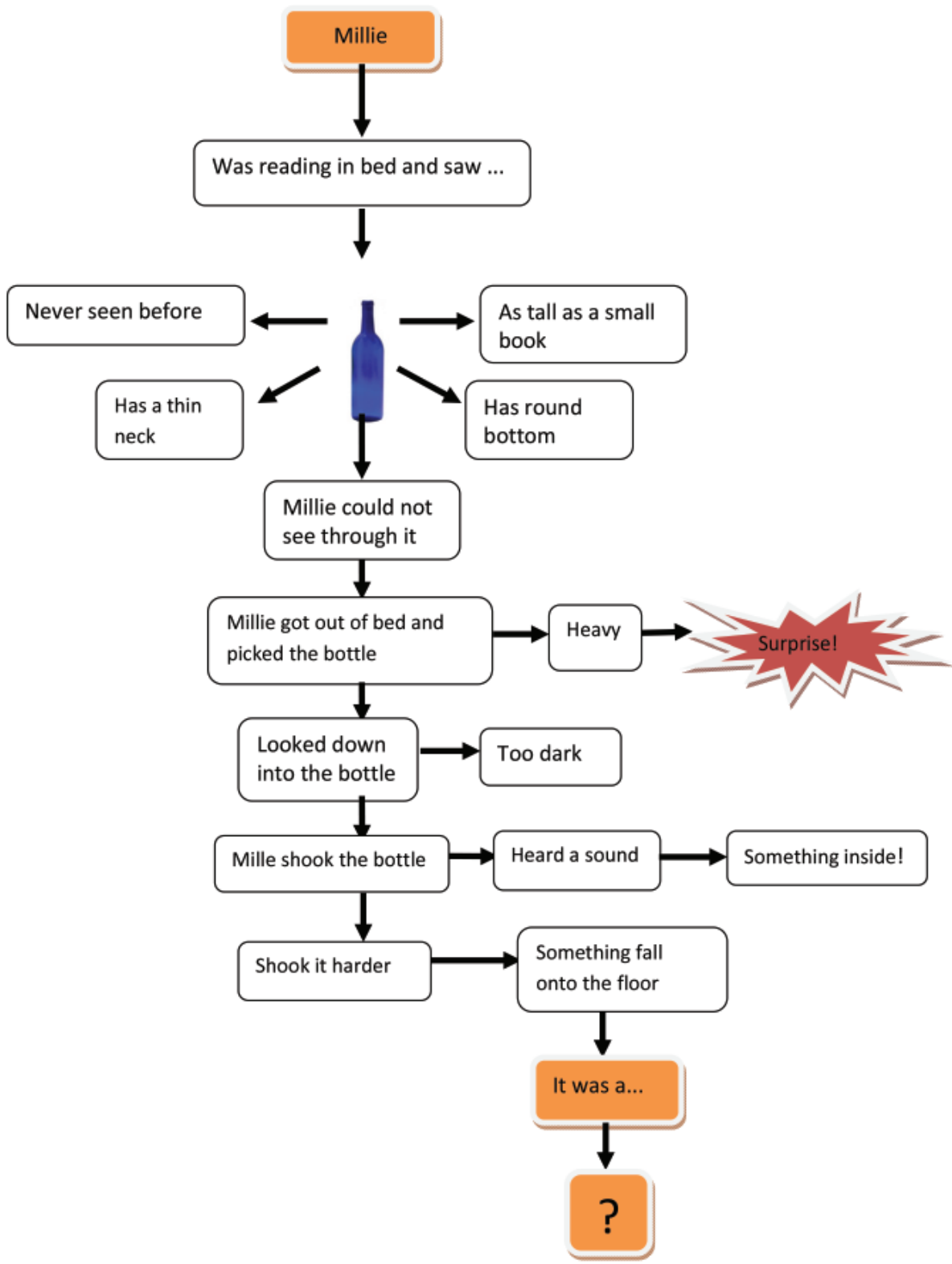